\title{
Relating the Farrell Nil-groups to the Waldhausen Nil-groups
}

\author{
Jean-François Lafont and Ivonne J. Ortiz \\ (Communicated by Andrew Ranicki)
}

\begin{abstract}
Every virtually cyclic group $\Gamma$ that surjects onto the infinite dihedral group $D_{\infty}$ contains an index two subgroup $\Pi$ of the form $H \rtimes_{\alpha} \mathbb{Z}$. We show that the Waldhausen Nil-group of $\Gamma$ vanishes if and only if the Farrell Nil-group of $\Pi$ vanishes.
\end{abstract}

2000 Mathematics Subject Classification: 19D35.

\section{Statement of results}

The Bass Nil-groups, Farrell Nil-groups, and Waldhausen Nil-groups appear respectively as pieces in the computation of the algebraic $K$-theory of direct products, semidirect products, and amalgamations. While the Bass Nil-groups have been extensively studied, much less is known for both the Farrell Nil-groups and the Waldhausen Nilgroups. For the purposes of computing the algebraic $K$-theory of infinite groups, the Nil-groups of virtually cyclic groups yield obstructions to certain assembly maps being isomorphisms. In particular, the vanishing/non-vanishing of Nil-groups is of crucial importance for computational aspects of algebraic $K$-theory. In this short note we prove the following result:

Main Theorem. Let $\Gamma$ be a virtually cyclic group that surjects onto the infinite dihedral group $D_{\infty}$, and $\Gamma=G_{1} *_{H} G_{2}$ be the corresponding splitting of groups (with $H$ of index two in both $G_{1}$ and $G_{2}$ ). Let $\Pi=H \rtimes_{\alpha} \mathbb{Z} \leq \Gamma$ be the canonical subgroup of $\Gamma$ of index two, obtained by taking the pre-image of the canonical index two $\mathbb{Z}$ subgroup of $D_{\infty}$. Then for $i=0,1$, the following two statements are equivalent:

(A) The Waldhausen Nil-group $N K_{i}\left(\mathbb{Z} H ; \mathbb{Z}\left[G_{1}-H\right], \mathbb{Z}\left[G_{2}-H\right]\right)$ for the group $\Gamma=$ $G_{1} *_{H} G_{2}$ vanishes.

(B) The Farrell Nil-group $N K_{i}(\mathbb{Z} H, \alpha)$ for the group $\Pi=H \rtimes_{\alpha} \mathbb{Z}$ vanishes.

The proof of our Main Theorem will be completed in Section 2, with some concluding remarks in Section 3.

Next, let us recall that the Farrell-Jones Isomorphism Conjecture for a finitely generated group $\Gamma$ states that the assembly map: 


$$
H_{n}^{\Gamma}\left(E_{\mathscr{V} \mathscr{C}}(\Gamma) ; \mathbb{K Z}^{-\infty}\right) \rightarrow H_{n}^{\Gamma}\left(E_{\mathscr{A} \mathscr{L} \mathscr{L}}(\Gamma) ; \mathbb{K}^{-\infty}\right)=K_{n}(\mathbb{Z} \Gamma)
$$

is an isomorphism. The term on the left is the generalized equivariant homology theory of the space $E_{\mathscr{V} \mathscr{C}}(\Gamma)$ with coefficients in the integral $K$-theory spectrum, where the space $E_{\mathscr{V} \mathscr{C}}(\Gamma)$ is a classifying space for $\Gamma$-actions with isotropy in the family $\mathscr{V} \mathscr{C}$ of virtually cyclic subgroups. The term on the right gives the algebraic $K$-theory of the integral group ring of $\Gamma$.

Explicit models for the classifying space $E_{\mathscr{V} \mathscr{C}}(\Gamma)$ are known for few classes of groups: virtually cyclic groups (take $E_{\mathscr{V} \mathscr{C}}(\Gamma)$ to be a point with trivial action), crystallographic groups (by work of Alves and Ontaneda [AO06]), hyperbolic groups (by work of Juan-Pineda and Leary [JL], and Lück [Lu05]), and in the case of relatively hyperbolic groups (due to the authors [LO]). In contrast, explicit models are known for $E_{\mathscr{F} \mathscr{F} \mathcal{N}}(\Gamma)$ for many classes of groups (see [Lu05] for a thorough survey), where $E_{\mathscr{F} \mathscr{F} \mathcal{N}}(\Gamma)$ is a classifying space for $\Gamma$-actions with isotropy in the family of finite subgroups. Furthermore, the algebraic $K$-theory of finite groups is much better understood than the algebraic $K$-theory of virtually cyclic groups. As such, it is interesting to know whether one can further reduce the computation of the algebraic $K$-theory of the integral group ring of $\Gamma$ to the computation of $H_{n}^{\Gamma}\left(E_{\mathscr{F} \mathscr{A} \mathcal{N}}(\Gamma) ; \mathbb{K Z}^{-\infty}\right)$, i.e., whether the natural map:

$$
H_{n}^{\Gamma}\left(E_{\mathscr{F} \mathscr{I} \mathcal{N}}(\Gamma) ; \mathbb{K Z}^{-\infty}\right) \rightarrow H_{n}^{\Gamma}\left(E_{\mathscr{V} \mathscr{C}}(\Gamma) ; \mathbb{K} \mathbb{Z}^{-\infty}\right)
$$

is an isomorphism. There is a well known criterion for this, namely the map will be an isomorphism for all $n \leq q$ if and only if, for every infinite virtually cyclic subgroup $Q$ of $\Gamma$, the map:

$$
H_{n}^{Q}\left(E_{\mathscr{F} \mathscr{T} \mathcal{N}}(Q) ; \mathbb{K} \mathbb{Z}^{-\infty}\right) \rightarrow K_{n}(\mathbb{Z} Q)
$$

is an isomorphism for all $n \leq q$ (see [DL98, Theorem 2.3] for $q<\infty$, and [FJ93, Theorem A.10] for $q=\infty)$. Since the cokernel of these maps are precisely the Nilgroups, our main theorem now gives us the following

Corollary. Let $\Gamma$ be a finitely generated group, and $q=0$ or 1 . Then the following two statements are equivalent:

- the relative assembly map

$$
H_{n}^{\Gamma}\left(E_{\mathscr{F} \mathscr{I} \mathcal{N}}(\Gamma) ; \mathbb{K} \mathbb{Z}^{-\infty}\right) \rightarrow H_{n}^{\Gamma}\left(E_{\mathscr{V} \mathscr{C}}(\Gamma) ; \mathbb{K} \mathbb{Z}^{-\infty}\right)
$$

is an isomorphism for $n \leq q$,

- for every subgroup $H \rtimes_{\alpha} \mathbb{Z} \leq \Gamma$ with $H$ finite, and for every $n \leq q$, the Farrell Nilgroup $N K_{n}(\mathbb{Z} H, \alpha)$ for the group $H \rtimes_{\alpha} \mathbb{Z}$ vanishes.

If in addition, we know that the Farrell-Jones Isomorphism Conjecture holds for the group $\Gamma$, then we have that (for $n \leq q)$ :

$$
H_{n}^{\Gamma}\left(E_{\mathscr{F} \mathscr{S} \mathcal{N}}(\Gamma) ; \mathbb{K Z}^{-\infty}\right) \cong K_{n}(\mathbb{Z} \Gamma) .
$$


Proof. We start by recalling that Farrell-Jones [FJ95] have shown that the Nil-groups (Bass, Farrell, and Waldhausen type) always vanish for $n \leq-1$. So we focus on $n=0,1$.

The proof follows immediately from the discussion above: if one has an infinite virtually cyclic subgroup of the form $H \rtimes_{\alpha} \mathbb{Z}$ for which the Farrell Nil-group $N K_{n}(\mathbb{Z} H, \alpha) \neq 0$, then the criterion above tells us that the relative assembly map fails to be an isomorphism for $n$. Let us now argue for the converse. We know that every infinite virtually cyclic subgroup $Q$ of $\Gamma$ is either a semidirect product $H \rtimes_{\alpha} \mathbb{Z}$, or surjects onto the infinite dihedral group $D_{\infty}$. For groups of the first type, we have that the Farrell Nil-group vanish (by assumption). For groups of the second type, we know that there is an index two subgroup which is a semi-direct product of a finite group $H$ with $\mathbb{Z}$ (where $\mathbb{Z}$ acts on $H$ via some automorphism $\alpha$ ). Our main theorem says that if the Farrell Nil-group $N K_{n}(\mathbb{Z} H, \alpha)$ associated to the semi-direct product is zero (which holds by hypothesis), then the Waldhausen Nil-group associated to $V$ are likewise automatically zero. In particular, if the Farrell Nil-groups vanish for every infinite virtually cyclic subgroup of the form $H \rtimes_{\alpha} \mathbb{Z}$, then the Nil-groups vanish for every infinite virtually cyclic subgroup $Q$ in $\Gamma$. The criterion discussed above now implies that the relative assembly map is actually an isomorphism.

Acknowledgements. The results in this paper expand on a method used in a previous paper by the authors [LO]. The technique in that previous paper (and hence ultimately the technique in the present paper) was originally suggested to us by Tom Farrell. We would like to thank Tom for his suggestion, as well as for his friendly advice throughout the years.

In addition, we would like to thank J. Grunewald, D. Juan-Pineda, I. Leary, S. Prassidis, and M. Varisco for helpful comments on a preliminary draft of this paper.

The work in this paper was partly supported by the National Science Foundation under grant DMS-0606002.

\section{Proof of the Main Theorem}

In this section, we will provide a proof of the main theorem. The proof will make use of two maps to compare the $K$-theory of $\mathbb{Z} \Gamma$ with the $K$-theory of $\mathbb{Z} \Pi$ :

- the maps $\sigma_{*}: K_{i}(\mathbb{Z} \Pi) \rightarrow K_{i}(\mathbb{Z} \Gamma)$, functorially induced by the inclusion $\Pi \hookrightarrow \Gamma$, and the transfer maps $\sigma^{*}: K_{i}(\mathbb{Z} \Gamma) \rightarrow K_{i}(\mathbb{Z} \Pi)$ (see Farrell-Hsiang [FH78]). This will be used to establish $(\mathrm{A}) \Rightarrow(\mathrm{B})$.

- a map $\pi_{i} \mathscr{A}: \pi_{i} \mathscr{P}\left(E ; \rho_{E}\right) \rightarrow \pi_{i} \mathscr{P}(E)$ between suitably defined spectra of stable pseudo-isotopies (see Farrell-Jones [FJ95]). This will be used to establish (B) $\Rightarrow(\mathrm{A})$.

Recall that in the situation we are dealing with, the group $\Pi=H \rtimes_{\alpha} \mathbb{Z}$ is the canonical index two subgroup of the group $\Gamma=G_{1} *_{H} G_{2}$.

Another result we will need is that, as shown by Farrell-Hsiang [FH68], the group $K_{i}(\mathbb{Z} \Pi)$ can be expressed in the following form: 


$$
K_{i}\left(\mathbb{Z} H_{\alpha}[\mathbb{Z}]\right) \cong C \oplus N K_{i}(\mathbb{Z} H, \alpha) \oplus N K_{i}\left(\mathbb{Z} H, \alpha^{-1}\right)
$$

where $C$ is a suitable quotient (determined by the automorphism $\alpha$ ) of the group $K_{i-1}(\mathbb{Z} H) \oplus K_{i}(\mathbb{Z} H)$. On the other hand, a classic result of Waldhausen [W78a], [W78b] (as reformulated by Connolly-Prassidis [CP02]) expresses $K_{i}(\mathbb{Z} \Gamma)$ as:

$$
\begin{aligned}
& K_{i}\left(\mathbb{Z}\left[G_{1} *_{H} G_{2}\right]\right) \\
& \cong N K_{i}\left(\mathbb{Z} H ; \mathbb{Z}\left[G_{1}-H\right], \mathbb{Z}\left[G_{2}-H\right]\right) \oplus\left[\left(K_{i}\left(\mathbb{Z} G_{1}\right) \oplus K_{i}\left(\mathbb{Z} G_{2}\right)\right) / K_{i}(\mathbb{Z} H)\right] .
\end{aligned}
$$

We will first establish that $(\mathrm{A}) \Rightarrow(\mathrm{B})$ (in Section 2.1). We will then briefly recall a construction of Farrell-Jones [FJ95] of a stratified fiber bundle $\rho_{E}: E \rightarrow X$, for a suitably defined space $E$, and stratified control space $X$ (Section 2.2). We will also explain in that section the relevance of their result to what we are trying to establish. Next we shall use the topology of the spaces $\rho_{E}^{-1}(x)$ for $x \in X$, to gain information on the $E^{2}$-terms in the Quinn spectral sequence (see Section 2.3). Finally, we shall use the information we obtain concerning the spectral sequence to prove that $(\mathrm{B}) \Rightarrow(\mathrm{A})$ (Section 2.4). Throughout Sections 2.1-2.4, we will be working exclusively with the case $i=1$. We will complete the proof in Section 2.5 by explaining the required modifications needed to obtain the case $i=0$.

2.1. Vanishing of Waldhausen Nil forces vanishing of Farrell Nil. In order to show that $(\mathrm{A}) \Rightarrow(\mathrm{B})$, we first assume that the Waldhausen Nil-group $N K_{1}\left(\mathbb{Z} H ; \mathbb{Z}\left[G_{1}-H\right]\right.$, $\left.\mathbb{Z}\left[G_{2}-H\right]\right)=0$. Note that under this hypothesis, the decomposition in equation (2) reduces to:

$$
K_{1}\left(\mathbb{Z}\left[G_{1} *_{H} G_{2}\right]\right) \cong\left(K_{1}\left(\mathbb{Z} G_{1}\right) \oplus K_{1}\left(\mathbb{Z} G_{2}\right)\right) / K_{1}(\mathbb{Z} H)
$$

We now want to argue that the corresponding Farrell Nil-group $N K_{1}(\mathbb{Z} H, \alpha)$ is trivial. The key observation is contained in the following:

Lemma 2.1. The map $\sigma_{*}$ is injective on the subgroup $N K_{1}(\mathbb{Z} H, \alpha)$ in the decomposition (1) of the group $K_{1}(\mathbb{Z} \Pi)$.

Proof. This follows from [FH68, Proposition 20], which shows that the composite $\operatorname{map} \sigma^{*} \circ \sigma_{*}: K_{1}(\mathbb{Z} \Pi) \rightarrow K_{1}(\mathbb{Z} \Pi)$ has an explicit expression in terms of the decomposition of $K_{1}(\mathbb{Z} \Pi)$ given in equation (1) above: it maps an element in the $N K_{1}(\mathbb{Z} H, \alpha)$ summand to the sum of itself with the image of this element under the canonical isomorphism $\tau: N K_{1}(\mathbb{Z} H, \alpha) \rightarrow N K_{1}\left(\mathbb{Z} H, \alpha^{-1}\right)$. This can be seen as follows: in the short exact sequence

$$
0 \rightarrow \Pi \rightarrow \Gamma \rightarrow \mathbb{Z} / 2 \rightarrow 0
$$

the $\mathbb{Z} / 2$ acts on $\Pi=H \rtimes_{\alpha} \mathbb{Z}$ via the map $z \mapsto-z$ on the $\mathbb{Z}$ factor. But the Farrell Nil-groups $N K_{1}(\mathbb{Z} H, \alpha)$ and $N K_{1}\left(\mathbb{Z} H, \alpha^{-1}\right)$ are canonically associated to the sub- 
semirings $\mathbb{Z}\left[H \rtimes_{\alpha} \mathbb{Z}^{+}\right]$and $\mathbb{Z}\left[H \rtimes_{\alpha} \mathbb{Z}^{-}\right]$, where $\mathbb{Z}^{+}, \mathbb{Z}^{-}$refers to the non-negative and non-positive integers respectively. Since the action of $\mathbb{Z} / 2$ on $\Pi$ interchanges these two sub-semirings inside $\mathbb{Z} \Pi$, the induced action of the non-trivial element $g \in \mathbb{Z} / 2$ on the $K_{1}(\mathbb{Z} \Pi)$ interchanges the two summands $N K_{1}(\mathbb{Z} H, \alpha)$ and $N K_{1}\left(\mathbb{Z} H, \alpha^{-1}\right)$ via the canonical isomorphism $\tau$ (this map was precisely the one used by FarrellHsiang to see that these two Nil-groups are isomorphic). Now the composite map $\sigma^{*} \circ \sigma_{*}: K_{1}(\mathbb{Z} \Pi) \rightarrow K_{1}(\mathbb{Z} \Pi)$ is given by the following formula

$$
x \mapsto \Sigma_{g \in \mathbb{Z} / 2} g_{*}(x)
$$

where $g_{*}: K_{1}(\mathbb{Z} \Pi) \rightarrow K_{1}(\mathbb{Z} \Pi)$ is the map induced on the $K$-theory of $\mathbb{Z} \Pi$ via the action of $g$ on $\Pi$ (recall that $\Pi$ is normal in $\Gamma$ ). In the situation we are interested in, the discussion above implies that for $x \in N K_{1}(\mathbb{Z} H, \alpha)$, we have $\left(\sigma^{*} \circ \sigma_{*}\right)(x)=x+\tau(x)$, where $\tau: N K_{1}(\mathbb{Z} H, \alpha) \rightarrow N K_{1}\left(\mathbb{Z} H, \alpha^{-1}\right)$ is the canonical isomorphism. This implies that the composite map $\sigma^{*} \circ \sigma_{*}$ is injective on the $N K_{1}(\mathbb{Z} H, \alpha)$ summand, and hence the map $\sigma_{*}$ must likewise be injective, concluding the proof of the lemma.

In particular, in the decomposition of the group $K_{1}\left(\mathbb{Z}\left[G_{1} *_{H} G_{2}\right]\right)$, we have that the group $N K_{1}(\mathbb{Z} H, \alpha)$ injects into the group $\left(K_{1}\left(\mathbb{Z} G_{1}\right) \oplus K_{1}\left(\mathbb{Z} G_{2}\right)\right) / K_{1}(\mathbb{Z} H)$. Since the latter group is a finitely generated abelian group, this implies that the group $N K_{1}(\mathbb{Z} H, \alpha)$ is finitely generated. But Grunewald [G1, Theorem 2.5] and Ramos [R] independently showed that the groups $N K_{1}(\mathbb{Z} H, \alpha)$ are either trivial or infinitely generated. This forces $N K_{1}(\mathbb{Z} H, \alpha)$ to vanish, establishing $(\mathrm{A}) \Rightarrow(\mathrm{B})$.

2.2. Some results of Farrell-Jones. Farrell and Jones in [FJ95, Section 2] defined a 3dimensional stratified control space $X$, and constructed from the pair $\Pi \hookrightarrow \Gamma$ a stratified fiber bundle $E$ over $X$. The stratified control space $X$ contains a distinguished point $p$, and the stratified fiber bundle $\rho_{E}: E \rightarrow X$ in their construction has the following properties:

- $E$ is a closed manifold with $\pi_{1}(E) \cong \Gamma$,

- for every $x \in X$ satisfying $x \neq p$, the group $\pi_{1}\left(E_{x}\right)$ is a finite group,

- for the distinguished point $p \in X, \pi_{1}\left(E_{p}\right) \cong \Pi$.

where $E_{x}=\rho_{E}^{-1}(x)$.

The important result for our purposes is [FJ95, Theorem 2.6], establishing that the group homomorphism

$$
\pi_{i} \mathscr{A}: \pi_{i} \mathscr{P}\left(E ; \rho_{E}\right) \rightarrow \pi_{i} \mathscr{P}(E)
$$

is an epimorphism for every integer $i$. Here $\mathscr{P}(E)$ is the spectrum of stable topological pseudoisotopies on $E, \mathscr{P}\left(E, \rho_{E}\right)$ is the spectrum of those stable pseudoisotopies which are controlled over $X$ via $\rho_{E}$, and $\mathscr{A}$ is the 'assembly' map. 
Now the relevance to the situation we are considering is that, by results of Anderson and Hsiang [AH77, Theorem 3], the lower homotopy groups of the pseudoisotopy spectrum coincide (with a shift in dimension) with the lower algebraic $K$ theory of the integral group ring of the fundamental group of the space. In particular, we have that the right hand side of the map in (3) is given by:

$$
\pi_{j} \mathscr{P}(E)= \begin{cases}W h(\Gamma), & j=-1 \\ \tilde{K}_{0}(\mathbb{Z} \Gamma), & j=-2 \\ K_{j+2}(\mathbb{Z} \Gamma), & j \leq-3\end{cases}
$$

To understand the left hand side of the map given in (3), we recall that Quinn [Qu82, Theorem 8.7] constructed a spectral sequence $E_{s, t}^{n}$ abutting to $\pi_{s+t} \mathscr{P}\left(E ; \rho_{E}\right)$ with $E_{s, t}^{2}=H_{s}\left(X ;\left\{\pi_{t} \mathscr{P}\left(\rho_{E}\right)\right\}\right)$. Here $\left\{\pi_{q} \mathscr{P}\left(\rho_{E}\right)\right\}, q \in \mathbb{Z}$, denotes the stratified system of abelian groups over $X$ where the group above $x \in X$ is $\pi_{q} \mathscr{P}\left(\rho_{E}^{-1}(x)\right)$.

Note that by Anderson and Hsiang's result (see [AH77, Theorem 3]) mentioned above, we also have that for every $x$ :

$$
\pi_{j} \mathscr{P}\left(E_{x}\right)= \begin{cases}W h\left(\pi_{1}\left(E_{x}\right)\right), & j=-1 \\ \tilde{K}_{0}\left(\mathbb{Z} \pi_{1}\left(E_{x}\right)\right), & j=-2 \\ K_{j+2}\left(\mathbb{Z} \pi_{1}\left(E_{x}\right)\right), & j \leq-3\end{cases}
$$

where $E_{x}=\rho_{E}^{-1}(x)$.

2.3. $\boldsymbol{E}^{\mathbf{2}}$-terms in the Quinn spectral sequence. Let us now assume that (B) holds, and let us analyze the $E^{2}$-terms in the Quinn spectral sequence. Recall that in our situation, we have that the groups $\pi_{1}\left(E_{x}\right)$ are all finite, except at one distinguished point $p$ where $\pi_{1}\left(E_{p}\right) \cong \Pi$. In particular, since the lower algebraic $K$-groups of the integral group ring of a finite group are finitely generated, this implies that the groups $\pi_{j} \mathscr{P}\left(E_{x}\right)$ are finitely generated groups, except possibly over the distinguished point $p$.

We now focus on the distinguished point $p$, and recall that we are assuming that the Farrell Nil-group $N K_{1}(\mathbb{Z} H, \alpha)$ is trivial. Since the Nil-groups $N K_{1}(\mathbb{Z} H, \alpha)$ and $N K_{1}\left(\mathbb{Z} H, \alpha^{-1}\right)$ are canonically isomorphic (see [FH68, Proposition 20]), we conclude that the Nil-groups vanish. This implies, by the formula for $K_{1}(\mathbb{Z} \Pi)$ given in equation (1) that $K_{1}(\mathbb{Z} \Pi)=C$. Recall that $C$ is a suitable quotient (determined by the automorphism $\alpha$ ) of the group $K_{0}(\mathbb{Z} H) \oplus K_{1}(\mathbb{Z} H)$. Since $H$ is finite, we have that $K_{0}(\mathbb{Z} H)$ and $K_{1}(\mathbb{Z} H)$ are finitely generated, and it follows that $K_{1}(\mathbb{Z} \Pi)=C$ is finitely generated. Since $W h(\Pi)$ is a quotient of $K_{1}(\mathbb{Z} \Pi)$, this implies that the Whitehead group $W h(\Pi)$ is likewise finitely generated.

From the result of Anderson and Hsiang [AH77], this tells us that at the distinguished point $p$,

$$
\pi_{-1} \mathscr{P}\left(E_{p}\right)=W h\left(\pi_{1}\left(E_{p}\right)\right)=W h(\Pi)
$$

is finitely generated. Furthermore, by results of Farrell-Jones [FJ95, Corollary 1.3] we know that for $j \leq-4$, the groups 


$$
\pi_{j} \mathscr{P}\left(E_{p}\right)=K_{j+2}\left(\mathbb{Z} \pi_{1}\left(E_{p}\right)\right)=K_{j+2}(\mathbb{Z} \Pi)
$$

vanish, and that the group $\pi_{-3} \mathscr{P}\left(E_{p}\right)=K_{-1}\left(\mathbb{Z} \pi_{1}\left(E_{p}\right)\right)=K_{-1}(\mathbb{Z} \Pi)$ is finitely generated. We summarize the information we have so far concerning the homotopy groups $\pi_{j} \mathscr{P}\left(E_{x}\right)$ in the following:

Fact 1. For the distinguished point $p \in X$, we have that for $j \leq-1$, the groups $\pi_{j} \mathscr{P}\left(E_{p}\right)$ are finitely generated, except possibly for the case where $j=-2$. For all other points $x \neq p$ in $X$, the groups $\pi_{j} \mathscr{P}\left(E_{x}\right)$ are finitely generated for all $j \leq-1$.

Now in terms of the stratified space $X$, let us recall how the $E_{p, q}^{2}$ term of the spectral sequence can be computed. The space $X$ is a stratified 3-dimensional CW-complex, with six cells. Furthermore, for points $x, y \in X$ lying in the interior of the same strata $\sigma^{p}$, we have that $E_{x}$ is homeomorphic to $E_{y}$ (we will denote this common space by $E_{\sigma^{p}}$ ). In particular, the stratified system of abelian groups is constant on the interior of each cell. Then the $E_{p, q}^{2}$ term is given by the homology of the chain complex:

$$
\cdots \rightarrow \bigoplus_{\sigma^{p+1}} \pi_{q} \mathscr{P}\left(E_{\sigma^{p+1}}\right) \rightarrow \bigoplus_{\sigma^{p}} \pi_{q} \mathscr{P}\left(E_{\sigma^{p}}\right) \rightarrow \bigoplus_{\sigma^{p-1}} \pi_{q} \mathscr{P}\left(E_{\sigma^{p-1}}\right) \rightarrow \cdots
$$

where each sum is over all appropriate dimensional strata in the decomposition of $X$. Note that from this chain complex, and the fact that $X$ is 3-dimensional, we immediately see that $E_{p, q}^{2}=0$ as soon as $p \leq-1$ or $4 \leq p$. Similarly, since all the groups in the chain complex are trivial for $q \leq-4$, we see that for such values of $q, E_{p, q}^{2}=0$. This gives us:

Fact 2. The only $E_{p, q}^{2}$-terms that can be non-zero are those for which $0 \leq p \leq 3$ and $-3 \leq q$. In particular, the spectral sequence collapses (by the $E^{4}$-stage).

Next we observe that the distinguished point $p \in X$ is actually a 0 -cell in the CWcomplex structure on $X$. From Fact 1 above, along with the chain complex describing the $E^{2}$-terms, we immediately obtain the following:

Fact 3. Within the range $q \leq-1$, all the $E^{2}$-terms in the spectral sequence are finitely generated, with the possible exception of the $E_{0,-2}^{2}$ term.

2.4. Vanishing of Farrell Nil forces vanishing of Waldhausen Nil. Now that we have gathered together information on the $E^{2}$-terms of the spectral sequence (assuming statement (B) holds), let us show that statement (A) must also hold.

Observe that, in an arbitrary spectral sequence, if a term $E_{p, q}^{2}$ is finitely generated, then $E_{p, q}^{k}$ is finitely generated for all $k \geq 2$. Furthermore, we have that if a term $E_{p, q}^{2}=0$, then $E_{p, q}^{k}=0$ for all $k \geq 2$. From Fact 2, our sequence collapses by the $E^{4}$-term, and we see that the only possible non-zero terms satisfying $p+q=-1$ are $E_{0,-1}^{4}, E_{1,-2}^{4}$, and $E_{2,-3}^{4}$. Combining Fact 3 with the observation above, we see 
that $\pi_{-1} \mathscr{P}\left(E ; \rho_{E}\right) \cong E_{0,-1}^{4} \oplus E_{1,-2}^{4} \oplus E_{2,-3}^{4}$ is finitely generated, and by Farrell-Jones [FJ95, Theorem 2.6], this group surjects onto $\pi_{-1} \mathscr{P}(E)=W h(\Gamma)$. In particular, we see that the group $W h(\Gamma)$ must be finitely generated, and hence the group $K_{1}(\mathbb{Z} \Gamma)$ is finitely generated. From the decomposition in equation (2), we see that the Waldhausen Nil-group $N K_{1}\left(\mathbb{Z} H ; \mathbb{Z}\left[G_{1}-H\right], \mathbb{Z}\left[G_{2}-H\right]\right)$ is a direct summand in $K_{1}(\mathbb{Z} \Gamma)$, and hence, must also be finitely generated. But Grunewald $[\mathrm{G} 1]$ has shown that the Waldhausen Nil-group is either trivial or infinitely generated. We conclude that the Waldhausen Nil-group is in fact trivial, completing the proof that $(\mathrm{B}) \Rightarrow(\mathrm{A})$.

2.5. Modifications for the case $\boldsymbol{i}=\mathbf{0}$. We now proceed to explain the modifications required to deal with the case $i=0$. The argument given above would extend verbatim, provided we had analogues for $i=0$ for the following key results (known to hold for $i=1)$ :

- the result in [FH68, Proposition 20], used in the proof of Lemma 2.1.

- the results of Ramos [R] and Grunewald [G1] establishing that the Waldhausen and Farrell Nil-groups are either trivial or infinitely generated.

To explain why these results extend to $i=0$, we recall some basic facts concerning the suspension functor from rings to rings. The cone ring $\Lambda \mathbb{Z}$ of $\mathbb{Z}$ is the ring of matrices over $\mathbb{Z}$ such that every column and every row contains only finitely many non-zero entries. The suspension ring $\Sigma \mathbb{Z}$ is the quotient of $\Lambda \mathbb{Z}$ by the ideal of finite matrices. For an arbitrary ring $R$ we define the suspension ring $\Sigma R=\Sigma \mathbb{Z} \otimes R$. The suspension fuctor $\Sigma \mathbb{Z} \otimes-$ has the key property that $K_{i}(R) \cong K_{i+1}(\Sigma R)$ for all $i \in \mathbb{Z}$.

Fact 4. For $\Pi \leq \Gamma$ as in our Main Theorem, with respect to the decomposition

$$
K_{0}\left(\mathbb{Z} H_{\alpha}[\mathbb{Z}]\right) \cong C \oplus N K_{0}(\mathbb{Z} H, \alpha) \oplus N K_{0}\left(\mathbb{Z} H, \alpha^{-1}\right)
$$

we have for all $x \in N K_{0}(\mathbb{Z} H, \alpha)$ the equality $\left(\sigma^{*} \circ \sigma_{*}\right)(x)=x+\tau(x)$, where $\tau: N K_{0}(\mathbb{Z} H, \alpha) \rightarrow N K_{0}\left(\mathbb{Z} H, \alpha^{-1}\right)$ is the canonical isomorphism.

To establish this fact, we merely note that [FH68, Proposition 20] holds for any ring $R$, and establishes that, for an abstract ring automorphism $\alpha$, we have (1) the $K_{1}$ of the twisted ring $R_{\alpha}[T]$ can be computed via

$$
K_{1}\left(R_{\alpha}[T]\right) \cong C \oplus N K_{1}(R, \alpha) \oplus N K_{1}\left(R, \alpha^{-1}\right),
$$

where $C$ is some suitable quotient of $K_{0}(R) \oplus K_{1}(R)$, and (2) the groups $N K_{1}(R, \alpha)$ and $N K_{1}\left(R, \alpha^{-1}\right)$ are canonically associated to the sub-semirings $R_{\alpha}\left[T^{+}\right]$and $R_{\alpha}\left[T^{-}\right]$. Here $T$ is an infinite cyclic group, $T^{+}$and $T^{-}$the semigroup generated by the generator and the inverse of the generator respectively.

Now observe that there is a canonical ring isomorphism $\Sigma\left(R_{\alpha}[T]\right) \cong(\Sigma R)_{1 \otimes \alpha}[T]$ (see [G2, Remark 2.12 part (2)]). This induces a commutative diagram: 


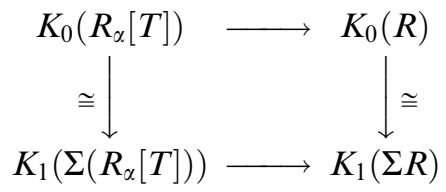

which implies that the kernel of the top row is isomorphic to the kernel of the bottom row. But these groups are, by definition, $N K_{0}(R, \alpha) \oplus N K_{0}\left(R, \alpha^{-1}\right)$ and $N K_{1}(\Sigma R, 1 \otimes \alpha) \oplus N K_{1}\left(\Sigma R, 1 \otimes \alpha^{-1}\right)$ respectively. Furthermore, the functor $\Sigma$ maps the sub-semiring $R_{\alpha}\left[T^{+}\right]$(and $R_{\alpha}\left[T^{-}\right]$) of the ring $R_{\alpha}[T]$ to the sub-semiring $(\Sigma R)_{1 \otimes \alpha}\left[T^{+}\right]$(and $(\Sigma R)_{1 \otimes \alpha}\left[T^{-}\right]$, respectively) of the ring $(\Sigma R)_{1 \otimes \alpha}[T]$. This forces the isomorphism above to restrict to isomorphisms $N K_{0}(R, \alpha) \cong N K_{1}(\Sigma R, 1 \otimes \alpha)$ and $N K_{0}\left(R, \alpha^{-1}\right) \cong N K_{1}\left(\Sigma R, 1 \otimes \alpha^{-1}\right)$ respectively. Finally, under the situation we are dealing with, we note that the non-trivial element $g \in \mathbb{Z} / 2=\Gamma / \Pi$ which acts on $R=\mathbb{Z} \Pi$ by interchanging the two sub-semirings $R_{\alpha}\left[T^{+}\right]$and $R_{\alpha}\left[T^{-}\right]$commutes with the functor $\Sigma$, and hence acts on $(\Sigma R)_{1 \otimes \alpha}[T]$ by permuting the two sub-semirings $(\Sigma R)_{1 \otimes \alpha}\left[T^{+}\right]$and $(\Sigma R)_{1 \otimes \alpha}\left[T^{-}\right]$. This implies that the map $\tau$ acts on the decomposition of $K_{0}(\mathbb{Z} \Pi)$ by interchanging the two factors $N K_{0}(R, \alpha)$ and $N K_{0}\left(R, \alpha^{-1}\right)$, as was required in the argument of Lemma 2.1. This completes the verification of the first point mentioned above.

For the second point mentioned above, we note that, by the argument in the previous paragraph, we have that $N K_{0}(\mathbb{Z} H, \alpha) \cong N K_{1}(\Sigma(\mathbb{Z} H), 1 \otimes \alpha)$. Since Grunewald [G1] showed that the $N K_{1}(R, \beta)$ is either trivial or infinitely generated, for any ring $R$ and any automorphism $\beta$ of finite order, we immediately have the corresponding property for $N K_{0}$. A similar argument shows that, for the Waldhausen Nil-groups, we have isomorphisms:

$$
\begin{aligned}
& N K_{0}\left(\mathbb{Z} H ; \mathbb{Z}\left[G_{1}-H\right], \mathbb{Z}\left[G_{2}-H\right]\right) \\
& \quad \cong N K_{1}\left(\Sigma(\mathbb{Z} H) ; \Sigma\left(\mathbb{Z}\left[G_{1}-H\right]\right), \Sigma\left(\mathbb{Z}\left[G_{2}-H\right]\right)\right)
\end{aligned}
$$

Note in the above expression that the functor $\Sigma$ has a natural extension to left bimodules, in the sense that if $B$ is a left bimodule for the ring $R$, then $\Sigma B$ is a left bimodule for $\Sigma R$ (see [G2, Section 2] for more details). Applying Grunewald's result for $N K_{1}$ to the right hand term, concludes the argument for the following:

Fact 5. For $\Pi \leq \Gamma$ as in our main theorem, we have that both

- the Farrell Nil-group $N K_{0}(\mathbb{Z} H, \alpha)$, and

- the Waldhausen Nil-group $N K_{0}\left(\mathbb{Z} H ; \mathbb{Z}\left[G_{1}-H\right], \mathbb{Z}\left[G_{2}-H\right]\right)$,

are either trivial or infinitely generated.

Finally, we explain how Facts 4 and 5 can be used to promote the arguments in Sections 2.1-2.4. Using Fact 4, the proof in Lemma 2.1 extends verbatim to show that the map $\sigma_{*}: K_{0}(\mathbb{Z} \Pi) \rightarrow K_{0}(\mathbb{Z} \Gamma)$ is injective on the subgroup $N K_{0}(\mathbb{Z} H, \alpha)$. Using 
Fact 5 and the decompositions of $K_{0}(\mathbb{Z} \Pi)$ and $K_{0}(\mathbb{Z} \Gamma)$ given in equations (1) and (2), the arguments in the last paragraph of Section 2.1 yield the implication $(\mathrm{A}) \Rightarrow(\mathrm{B})$ for the case $i=0$.

In the proof of the converse, the sole changes occur in Section 2.3, Fact 1, where all the groups $\pi_{j} \mathscr{P}\left(E_{p}\right)$ are finitely generated except possibly for $j=-1$ (instead of $j=-2$ ). Correspondingly, there is a change in Fact 3, where within the range $q \leq-1$, all the $E^{2}$-terms of the spectral sequence are finitely generated except possibly for the $E_{0,-1}^{2}$ term. The argument in Section 2.4 allows one to conclude that the group $\pi_{-2} \mathscr{P}\left(E ; \rho_{E}\right) \cong E_{0,-2}^{4} \oplus E_{1,-3}^{4}$ is finitely generated, and the rest of the discussion in Section 2.4, along with Fact 5, completes the converse implication $(\mathrm{B}) \Rightarrow(\mathrm{A})$ for the case $i=0$.

\section{Concluding remarks}

While our main theorem provides a relationship between the Farrell Nil-groups and the Waldhausen Nil-groups, one can speculate about whether one can establish a further reduction to the Bass Nil-groups. This motivates the following:

Question. For an arbitrary ring $R$, are the following two statements equivalent:

- the Bass Nil-group $N K_{i}(R)$ vanishes, and

- the Farrell Nil-groups $N K_{i}(R, \alpha)$ vanish for every automorphism $\alpha$ of the ring $R$.

This last question seems extremely difficult to answer. The most interesting case would be where the ring $R$ is the integral group ring of a finitely generated group $G$, and the automorphisms are generated by automorphisms of $G$.

Finally, we point out that if one does not assume vanishing of the Farrell Nil-group of $\Pi$, the Quinn spectral sequence still gives us some information relating the Farrell Nil-group of $\Pi$ with the Waldhausen Nil-group of $\Gamma$. Indeed, a more careful analysis of the Quinn spectral sequence can be used to show that the Farrell Nil-group appearing in the $E^{2}$ term survives to the $E^{4}$ stage, and surjects onto the Waldhausen Nil-group (see Prassidis [P97, pages 412-413] for a similar analysis). This is motivated by the well-known philosophy that, in the map of Farrell-Jones:

$$
\pi_{i} \mathscr{A}: \pi_{i} \mathscr{P}\left(E ; \rho_{E}\right) \rightarrow \pi_{i} \mathscr{P}(E)
$$

the controlled (resp. non-controlled) part of the pseudo-isotopy spectrum $\mathscr{P}\left(E ; \rho_{E}\right)$ must map to the controlled (resp. non-controlled) part of the pseudo-isotopy spectrum $\mathscr{P}(E)$. The non-controlled part is precisely the Nil-groups.

Now one could use this surjection to show that the exponent of the Waldhausen Nil-group must divide the exponent of the Farrell Nil-group. We observe however that the estimates this would yield provide no improvement on known estimates (due independently to Grunewald [G1] and Ramos [R]). In the interest of clarity of exposition, the authors have chosen to omit this further analysis, leaving the details to the interested reader. 


\section{References}

[AO06] Alves A. and Ontaneda P.: A formula for the Whitehead group of a three-dimensional crystallographic group. Topology 45 (2006), 1-25

[AH77] Anderson D. R. and Hsiang W. C.: The functors $K_{-i}$ and pseudo-isotopies of polyhedra. Ann. of Math. 105 (1977), 210-223

[Bas68] Bass H.: Algebraic K-theory. W. A. Benjamin, New York 1968

[CP02] Connolly F. X. and Prassidis S.: On the exponent of the cokernel of the forget-control map on $K_{0}$-groups. Fund. Math. 172 (2003), 201-216

[DL98] Davis J. F. and Lück W.: Spaces over a category and assembly maps in isomorphism conjectures in $K$ - and $L$-theory. $K$-theory 15 (1998), 201-252

[F77] Farrell F. T.: The nonfiniteness of Nil. Proc. Amer. Math. Soc. 65 (1977), 215-216

[FJ93] Farrell F. T. and Jones L.: Isomorphism conjectures in algebraic $K$-theory. J. Amer. Math. Soc. 6 (1993), 249-297

[FJ95] Farrell F. T. and Jones L.: The lower algebraic $K$-theory of virtually infinite cyclic groups. $K$-theory 9 (1995), 13-30

[FH68] Farrell F. T. and Hsiang W. C.: A formula for $K_{1} R_{\alpha}[T]$, 1970 Applications of Categorical Algebra (Proc. Sympos. Pure Math. 17), New York (1968), 192-218

[FH78] Farrell F. T. and Hsiang W. C.: The topological-Euclidean space form problem. Inventiones Math. 45 (1978), 181-192

[G1] Grunewald J.: Non-finiteness results for Nil-groups, preprint (2006)

[G2] Grunewald J.: The behavior of Nil-Groups under localization and the relative assembly map, preprint (2006)

[JL] Juan-Pineda D. and Leary I. J.: On classifying spaces for the family of virtually cyclic subgroups; to appear in Recent Developments in Algebraic Topology (Contemp. Math. 407). Amer. Math. Soc. 2006

[LO] Lafont J. F. and Ortiz I. J.: Relative hyperbolicity, classifying spaces, and lower algebraic $K$-theory, preprint (2006)

[Lu05] Lück W.: Survey on classifying spaces for families of subgroups. In: Infinite groups: geometric, combinatorial and dynamical aspects (Progr. Math. 248), 269-322. Birkhäuser, Basel 2005

[P97] Prassidis S.: A split exact sequence of equivariant $K$-groups of virtually nilpotent groups. $K$-theory 11 (1997), 397-415

[Qu82] Quinn F.: Ends of maps II. Invent. Math. 68 (1982), 353-424

[R] Ramos R.: Non finiteness of twisted Nils, preprint (2006)

[W78a] Waldhausen F.: Algebraic $K$-theory of generalized free products. I, II. Ann. of Math. (2) 108 (1978), 135-204

[W78b] Waldhausen F.: Algebraic $K$-theory of generalized free products. III, IV. Ann. of Math. (2), 108 (1978), 205-256

Received 5 August 2006; in final form 25 September 2006

Department of Mathematics, Ohio State University, Columbus, OH 43210

jlafont@math.ohio-state.edu

Department of Mathematics and Statistics, Miami University, Oxford, OH 45056

ortizi@muohio.edu 